\title{
Induction and repression of $\alpha$-amylase production in batch and continuous cultures of Aspergillus oryzae
}

\author{
Rikke Mørkeberg, Morten Carlsen and Jens Nielsen
}

Center for Process

Biotechnology.

Department of

Biotechnology, Block 223,

Technical University of

Denmark, DK-2800 Lyngby, Denmark

\author{
Author for correspondence: Morten Carlsen. Tel: +45 45252659. Fax: +4545884148. \\ e-mail: MC@IBT.DTU.DK
}

\begin{abstract}
The intra- and extracellular concentrations of $\alpha$-amylase in Aspergillus oryzae have been measured during batch culture of a wild-type strain and two recombinant strains. The mean intracellular level for the two recombinant strains was about four to five times the level of the wild-type strain. The recombinant strains also had a higher $\alpha$-amylase productivity, whereas the residence time of the intracellular $\alpha$-amylase pool was approximately the same for the three strains. At high glucose concentrations there was a low constitutive synthesis of $\alpha$-amylase, whereas at low glucose concentrations derepression resulted in an increased production rate. Shifts from a glucose- to a maltose-limited chemostat showed that maltose induces both the production and secretion of $\alpha$-amylase. Finally, from immunoblots, both a glycosylated and an unglycosylated $\alpha$-amylase have been detected.
\end{abstract}

Keywords: Aspergillus oryzae, batch culture, continuous culture, $\alpha$-amylase, intracellular measurements

\section{INTRODUCTION}

A large number of industrial enzymes are derived from filamentous fungi belonging to the genus Aspergillus. It is a characteristic of these fungi that they are common and mostly harmless, and that they secrete a number of extracellular enzymes in relatively large quantities.

In this study $\alpha$-amylase production by Aspergillus oryzae is considered. $A$. oryzae has been used in Asian food preparation for thousands of years. Recently, it has been shown that $A$. oryzae exhibits potential as a host for the production of many different heterologous proteins, both proteins from other fungi (Christensen $e$ t al., 1988; HugeJensen et al., 1989) and mammalian proteins (Ward et al., 1992; Tsuchiya et al., 1993). Today, A. oryzae is used industrially in the production of $\alpha$-amylase, proteases and the heterologous lipase from Humicola lanuginosa (Barbesgaard et al., 1992).

The mechanisms behind growth and product formation are critical in the optimization of a production process. Despite many years of experience with the culture of $A$. oryzae, these mechanisms are poorly understood and only a few attempts have been made to analyse the regulation

Abbreviations: AMG, amyloglycosidase; FAU, $\alpha$-amylase activity unit defined as the amount of $\alpha$-amylase that hydrolyses $5.26 \mathrm{~g}$ starch at $37^{\circ} \mathrm{C}$ in $1 \mathrm{~h}$; FIA, Flow Injection Analysis; PNGase F, N-glycosidase F of the production of $\alpha$-amylase in $A$. oryzae. Yabuki et al. (1977) and Erratt et al. (1984) showed that $\alpha$-amylase production in $A$. oryzae is induced by maltose and starch, respectively, and other studies with the related fungus $A$. awamori have indicated glucose repression on the production of $\alpha$-amylase (Bhella \& Altosaar, 1987). Common to these experiments, however, is the fact that they have all been carried out in shake flasks where the culture conditions are poorly controlled.

In this study the intra- and extracellular concentrations of $\alpha$-amylase were measured throughout cultivation in bioreactors, with the aim of investigating the influence of the substrates glucose and maltose on $\alpha$-amylase production by $A$. oryzae. $\alpha$-Amylase is a starch-degrading enzyme, which catalyses the hydrolysis of internal $\alpha(1-4)$ glucosidic bonds in amylose, amylopectin and glycogen, but is not able to attack $\alpha(1-6)$-glucosidic linkages in the branched amylopectin and glycogen. $\alpha$-Amylase comprises a single polypeptide chain with 478 amino acid residues and one $N$-linked carbohydrate side-chain (McKelvy \& Lee, 1969).

\section{METHODS}

Organism and culture conditions. The three strains of $A$. oryzae were obtained from Novo Nordisk. The wild-type strain (A1560) originated from wild-type strain IFO4177 from the Institute for Fermentation, Osaka, Japan. Strain CF1.1 is a 
transformant of A1560 containing additional copies of the $\alpha$ amylase gene. The third strain, CF2.1, is a morphological mutant of CF1.1 that was isolated after treatment of CF1.1 spores with nitrosoguanidine (NTG).

Conidia from 6-8-d-old cultures (Carlsen, 1994) were inoculated into a defined batch medium containing $\left(1^{-1}\right): 25 \cdot 0 \mathrm{~g}$ glucose (monohydrate), $7.3 \mathrm{~g} \quad\left(\mathrm{NH}_{4}\right)_{2} \mathrm{SO}_{4}, 1.5 \mathrm{~g} \quad \mathrm{KH}_{2} \mathrm{PO}_{4}, 1.0 \mathrm{~g}$ $\mathrm{MgSO}_{4} .7 \mathrm{H}_{2} \mathrm{O}, 1.0 \mathrm{~g} \mathrm{NaCl}, 0.1 \mathrm{~g} \mathrm{CaCl} .2 \mathrm{H}_{2} \mathrm{O}, 0.5 \mathrm{ml}$ trace metal solution (14.3 $\mathrm{g} \mathrm{ZnSO}_{4} \cdot 7 \mathrm{H}_{2} \mathrm{O}, 2.5 \mathrm{~g} \mathrm{CuSO}_{4} \cdot 5 \mathrm{H}_{2} \mathrm{O}, 0.5 \mathrm{~g}$ $\mathrm{NiCl}_{2} \cdot 6 \mathrm{H}_{2} \mathrm{O}$ and $\left.13.8 \mathrm{~g} \mathrm{FeSO} \cdot 7 \mathrm{H}_{2} \mathrm{O}\right)$. The medium for continuous culture was identical to the batch medium except for the carbon and nitrogen content $\left[8.0 \mathrm{~g}\right.$ glucose monohydrate $\mathrm{l}^{-1}$ and $5.0 \mathrm{~g}\left(\mathrm{NH}_{4}\right)_{2} \mathrm{SO}_{4} \mathrm{l}^{-1}$.

All batch cultures were carried out in a 15 I MBR bioreactor and continuous cultures in a 71 Chemap-bioreactor. The temperature was $30^{\circ} \mathrm{C}$ and the $\mathrm{pH}$ was kept constant at 6.0 by adding $2 \mathrm{M} \mathrm{H}_{2} \mathrm{SO}_{4}$ or $4 \mathrm{M} \mathrm{NaOH}$ as necessary. Aeration was kept constant at 1 vol. air (vol. medium) $)^{-1} \mathrm{~min}^{-1}$. The dissolved oxygen tension was kept above $60 \%$ by controlling the agitation rate. The biomass concentration was measured by filtering the sample on a dry and preweighed filter, washing with $0.9 \%$ $\mathrm{NaCl}$, followed by drying at $105^{\circ} \mathrm{C}$ for $24 \mathrm{~h}$.

Measurement of glucose. Glucose concentration was measured in cell-free samples by a Flow Injection Analysis (FIA) system based on the enzymic oxidation of $\beta$-D-glucose to D-gluconolactone by immobilized glucose oxidase at $\mathrm{pH} 6.5$. Hydrogen peroxide generated in this oxidation is detected in the FIA system by oxidation of luminol, whereby light with a maximum intensity at $430 \mathrm{~nm}$ is emitted (Benthin et al., 1991 ; Carlsen et al., 1995a).

Extracellular $\alpha$-amylase measurements. A FIA system was used to measure the extracellular $\alpha$-amylase concentration. The principle is the decolorization of a sample containing a blue iodine-starch complex by the $\alpha$-amylase from the culture sample (Carlsen et al., 1994). The $\alpha$-amylase activity unit, FAU, is defined as the amount of $\alpha$-amylase that hydrolyses 5.26 g starch at $37^{\circ} \mathrm{C}$ in $1 \mathrm{~h}$ (Hansen, 1984). The FIA system measures the total amylolytic activity, but the exoacting glucoamylase activity does not give a substantial response by injection in the FIA system.

Preparation of intracellular $\alpha$-amylase samples. The sample preparation for intracellular $\alpha$-amylase measurements should be carried out as fast and as cold as possible to get a representative intracellular protein sample. The sample was therefore filtered rapidly in a cold Büchner funnel and washed with cold $0.9 \%$ $\mathrm{NaCl}$ solution, whereafter the biomass was instantly frozen using liquid nitrogen. The frozen biomass was stored at $-80^{\circ} \mathrm{C}$. Frozen biomass $(100 \mathrm{mg}$ ) was resuspended in $1.5 \mathrm{ml}$ $0 \cdot 1 \mathrm{M}$ sodium phosphate buffer, $\mathrm{pH} 5$, and kept on ice. The buffer contained the inhibitors phenanthroline $(10 \mathrm{mM}), \mathrm{PMSF}$ $(1 \mathrm{mM})$, and pepstatin $\mathrm{A}(3 \mu \mathrm{M})$ (all obtained from Sigma) to prevent unwanted proteolysis. Phenanthroline (and not the common EDTA) was used as metalloprotease inhibitor, since it has a much lower affinity for calcium which stabilizes $\alpha$-amylase against protease degradation (Stein \& Fischer, 1958).

The mycelium was disrupted in a Retsch bead-mill for $15 \mathrm{~min}$ with $0.5 \mathrm{ml}$ glass beads $(0.75-1.0 \mathrm{~mm})$ at $4{ }^{\circ} \mathrm{C}$. The samples were centrifuged (10 min, 15000 r.p.m., $4^{\circ} \mathrm{C}$ ) and the supernatant with the intracellular proteins was collected and stored at $-20^{\circ} \mathrm{C}$.

Quantification of intracellular $\alpha$-amylase. Intracellular samples were subjected to SDS-PAGE $(10 \%, \mathrm{w} / \mathrm{v}$, acrylamide containing $0 \cdot 25 \%$ bis-acrylamide, Nationals Diagnostics) according to the method described by Laemmli (1970). Following electrophoresis, the separated proteins in the gel were electro- phoretically transferred to a nitrocellulose membrane (pore size $0.45 \mu \mathrm{m}$ ), which was then incubated with polyclonal rabbit antibodies (Novo Nordisk) raised against the $A$. oryzae $\alpha$ amylase. The immuno-complex was detected with peroxidaseconjugated goat anti-rabbit antibodies (Dakopatts) and visualized by adding $\mathrm{H}_{2} \mathrm{O}_{2}$ and the hydrogen donor, 4-chloro-1naphthal (Sigma). This resulted in a blue precipitate on the blot.

Quantitative values of the amount of protein were obtained by measuring the intensity of the blue bands with a laser scanner (LKB UltroScan XL Densitometer). Four $\alpha$-amylase standards with a known amount of enzyme (1, 2, 4 and $10 \mathrm{mFAU})$ were added on each gel and used to generate a standard curve. Purified $\alpha$-amylase produced by $A$. oryzae (obtained from Novo Nordisk) was used as a standard. In all cases a linear standard curve was obtained with a correlation coefficient better than 0.99 and a relative SD of the slope below $5 \%$. The samples were diluted to be within the linear range of the standard curve.

Deglycosylation of $\alpha$-amylase. $N$-Glycosidase F (PNGase F, Sigma) which cleaves the $N$-glycan linkage of glycoproteins was used to deglycosylate $\alpha$-amylase. Sodium phosphate buffer $(20 \mu \mathrm{l}, 0 \cdot 1 \mathrm{M}, \mathrm{pH} 8)$ and $100 \mu \mathrm{g}$ protein were added to $20 \mu \mathrm{l}$ sample and incubated for $20 \mathrm{~h}$ at $37^{\circ} \mathrm{C}$. SDS-PAGE and immunoblotting of the samples followed.

Analysis of amylolytic activity. Erratt et al. (1984) described a method to assay $\alpha$-amylase activity and other starch-degrading activity in an SDS-gel. Following SDS-PAGE, the gel was washed for $10 \mathrm{~min}$ in distilled water. The staining procedure comprises three steps. Firstly, the gel was saturated with starch $\left(1 \%, \mathrm{w} / \mathrm{v}\right.$, starch solution); $2 \times 1 \mathrm{~h}$ at $60^{\circ} \mathrm{C}$ followed by $40 \mathrm{~min}$ at $4{ }^{\circ} \mathrm{C}$. Secondly, the gel was washed with distilled water and incubated for $2.5 \mathrm{~h}$ in a $0.15 \mathrm{M}$ sodium citrate/phosphate buffer, pH 4.8 (fresh buffer after $1.5 \mathrm{~h}$ ). Finally, the buffer was removed and the gel stained with a $\mathrm{KI} / \mathrm{I}_{2}$ solution $\left(10 \mathrm{~g} \mathrm{KI}, 1 \mathrm{~g} \mathrm{I}_{2}\right.$ in $100 \mathrm{ml}$ distilled water). Clear spots caused by amylolytic activity appeared in the blue gel.

\section{RESULTS}

\section{Identification of $\alpha$-amylase}

SDS-PAGE followed by immunoblotting was chosen to identify $\alpha$-amylase, since it specifically detects the protein of interest. This method also enables detection of more than one form of $\alpha$-amylase, e.g. preforms, unglycosylated and overglycosylated forms. By separating the proteins it is easy to distinguish them from each other and, furthermore, it is possible to estimate their molecular mass.

Fig. 1 shows an immunoblot where intra- and extracellular samples were loaded next to each other. The samples were from a glucose-grown batch culture of the wild-type strain A1560 (lanes 4 and 5) and the recombinant strain CF2.1 (lanes 2 and 3). Both intra- and extracellular samples from the two strains revealed two clear bands, a thick band at $54 \pm 2 \mathrm{kDa}$ and a thinner band at $66 \pm 2 \mathrm{kDa}$ (mean values $\pm S D$ for five replicates). The first value correlates very well with the reported molecular mass of purified $\alpha$-amylase from $A$. oryzae (Erratt et al., 1984; McKelvy \& Lee, 1969). From the amino acid sequence, $\alpha-$ amylase has been calculated to be $52.6 \mathrm{kDa}$, and since it contains a $1.5 \mathrm{kDa}$ carbohydrate chain, the molecular mass is $54 \cdot 1 \mathrm{kDa}$.

In the samples taken from cultures of the genetically manipulated strain, a third band was detected (Fig. 1, 


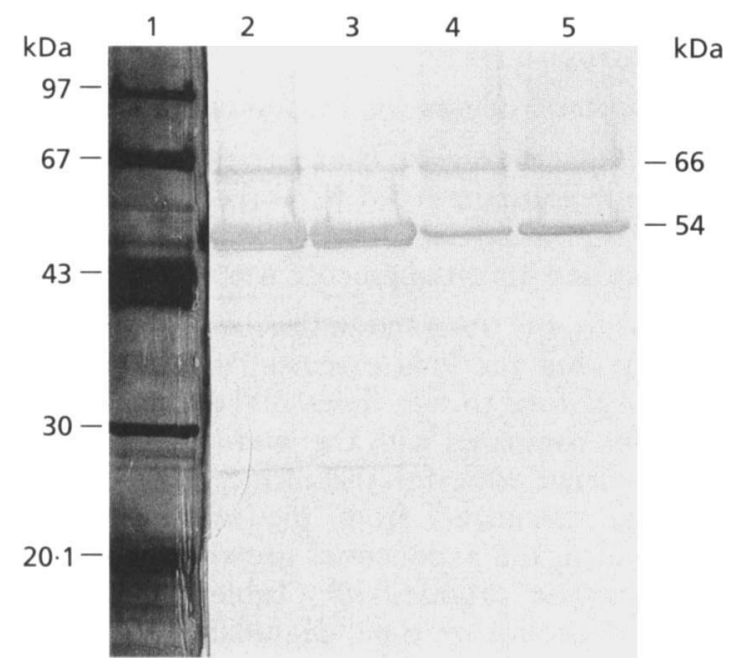

Fig. 1. Immunological identification of $\alpha$-amylase. Lanes: 1 , molecular mass markers; 2 and 3 , intra- and extracellular samples, respectively, from batch culture with the recombinant strain CF2.1; 4 and 5, intra- and extracellular samples, respectively, from batch culture with the wild-type strain A1560. The molecular mass markers were visualized by silver staining.

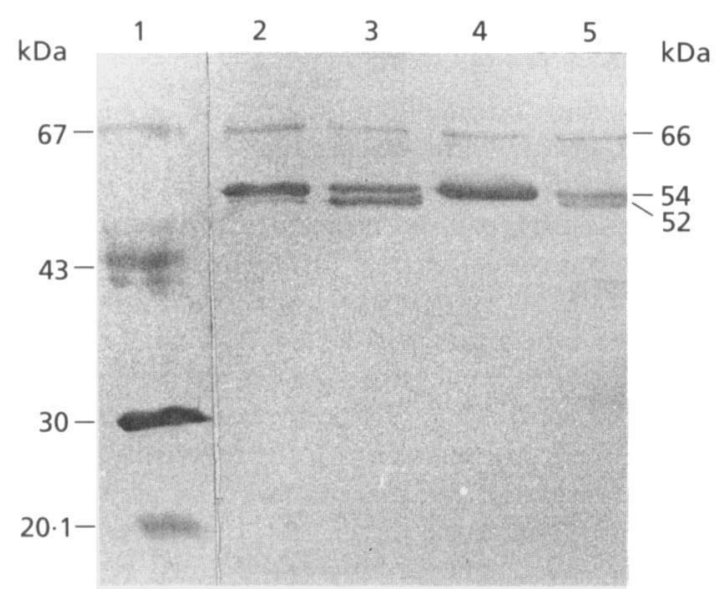

Fig. 2. Glycosylation of samples. Lanes: 1, molecular mass markers; 2, untreated CF1.1 sample; 3, CF2.1 sample deglycosylated by PNGase F; 4, untreated $\alpha$-amylase standard; $5, \alpha$-amylase standard deglycosylated by PNGase F. Note that the $66 \mathrm{kDa}$ band can also be seen in the $\alpha$-amylase standard, which indicates that the standard was not $100 \%$ pure. Proteins were visualized by the immuno technique described in Methods.

lanes 2 and 3). It is a weak band just below the $54 \mathrm{kDa}$ band and it was suspected to be the unglycosylated form of $\alpha$-amylase, which as mentioned above has a molecular mass of $52 \cdot 6 \mathrm{kDa}$.

\section{Deglycosylation}

To test whether the $52 \mathrm{kDa}$ band indeed represented an unglycosylated form of $\alpha$-amylase, deglycosylation of a

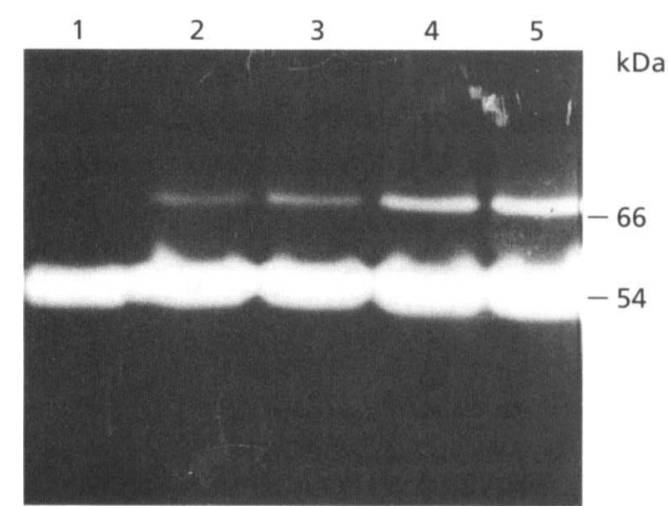

Fig. 3. Analysis of amylolytic activity. The figure shows an in situ activity gel. Lanes: $1, \alpha$-amylase standard; 2 and 3 , intraand extracellular samples, respectively, from batch culture with the wild-type strain A1560; 4 and 5, intra- and extracellular samples, respectively, from batch culture with the recombinant strain CF2.1. The molecular mass of the activity bands was determined by comparing a silver-stained molecular mass marker (separated from the gel after SDS-PAGE), taking into account that the in situ activity gel was 1.12 times longer than the silver-stained gel, because of the different treatment of the gels.

few samples was carried out. An intracellular sample of CF2.1 and the $\alpha$-amylase standard were deglycosylated with PNGase F, and the samples were loaded on a SDSgel together with untreated samples. As shown in Fig. 2, the $\alpha$-amylase band in the deglycosylated samples split up into two clear bands, which indicates a partial deglycosylation. This was further supported by intensity measurements of the bands, which showed that the sum of the intensity of the bands in lane 3 was the same as for the $54 \mathrm{kDa}$ band in lane 2 (Fig. 2). The deglycosylated form had the same position as the weak band of $52 \mathrm{kDa}$ in the untreated sample of CF2.1 and this band was therefore believed to represent the unglycosylated form of $\alpha$ amylase.

\section{Analysis of amylolytic activity}

Activity gels were carried out to determine the number of proteins with starch-degrading activity (Fig. 3). The gels showed two bands with starch-degrading activity at $54 \mathrm{kDa}$ and $66 \mathrm{kDa}$ for each analysed sample (both intraand extracellular samples from cultures of A1560 and CF2.1). The $\alpha$-amylase standard also showed the $66 \mathrm{kDa}$ band, but it is very weak and cannot be seen on the photograph. Only one other starch-degrading enzyme has been reported to be produced by $A$. oryzae; an exoacting amyloglycosidase (AMG) (Fogarty, 1983), and it was therefore believed that the $66 \mathrm{kDa}$ band represented this exoenzyme.

\section{Growth and $\alpha$-amylase activity in batch culture}

Three batch cultures were carried out on a glucose medium, one with each of the three strains. Glucose, biomass, and intra- and extracellular $\alpha$-amylase concentrations were measured during all three batch cultures, 

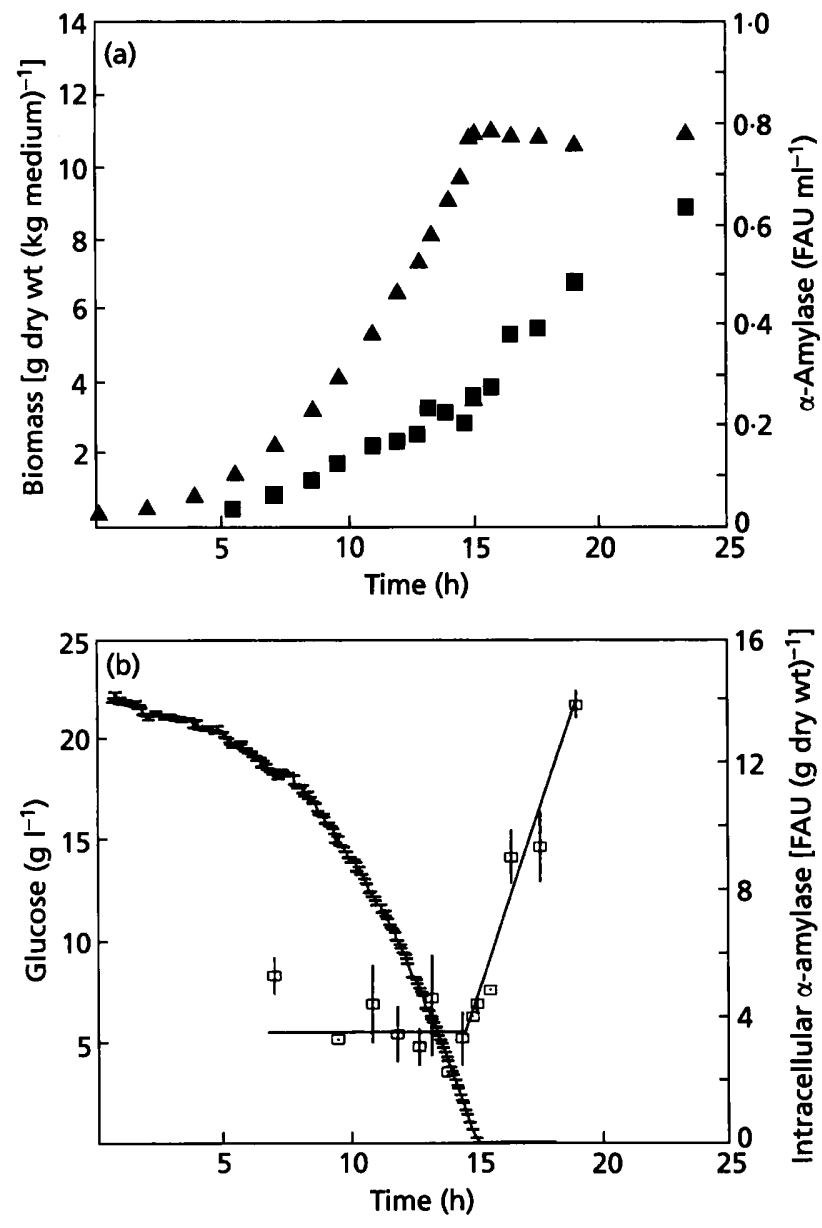

Fig. 4. (a) Batch culture with A1560. Biomass concentration (A) and extracellular $\alpha$-amylase activity ( $\square$ ) plotted in a linear plot against the batch culture time with A1560. (b) Glucose concentration measured on-line (-) and intracellular $\alpha$-amylase concentration ( $\square$ ) plotted against batch culture time with A1560. The absolute SD for the $\alpha$-amylase concentration is given as vertical error bars.

and the results for the wild-type strain A1560 are shown in Fig. 4.

Exponential growth occurred during the first $10 \mathrm{~h}$, followed by a decline in the specific growth rate. The $\alpha$ amylase concentration also increased exponentially at the same rate in this period. This shows that $\alpha$-amylase production is growth-related and the constant, specific rate of $\alpha$-amylase production is given in Table 1. After
$15 \mathrm{~h}$, the glucose was exhausted (on-line glucose measurement in Fig. 4b), but $\alpha$-amylase secretion continued to increase by a factor of 2 during the following 4 h (Fig. 4a).

The intracellular $\alpha$-amylase concentration (Fig. $4 \mathrm{~b}$ ) was approximately constant at $3.5 \mathrm{FAU}\left(\mathrm{g}_{\text {dry }} \mathrm{wt}\right)^{-1}$ during the first $15 \cdot 0 \mathrm{~h}$, but a drastic increase in the intracellular $\alpha-$ amylase occurred after the glucose was exhausted.

A similar trend was observed in the batch cultures of the other strains, but the final extracellular $\alpha$-amylase concentration was four to five times higher for the recombinant strains compared with the wild-type strain (Table 1). The residence time for the intracellular pool of $\alpha$ amylase was calculated from the mean intracellular concentration in the exponential growth phase and the specific $\alpha$-amylase productivity (Table 1 ). In the calculation of the residence time, degradation of $\alpha$-amylase was not considered since it has been shown to be very stable at pH 6.0 (Carlsen et al., 1995b).

\section{Continuous culture}

A continuous culture with the wild-type strain A1560 was carried out. The culture was started as a glucose-limited chemostat, and after $118.75 \mathrm{~h}$ the feed was shifted to a maltose medium. The shift in the carbon source did not give rise to any changes either in the $\mathrm{CO}_{2}$ and $\mathrm{O}_{2}$ measurements in the exhaust gas, or in the dry weight measurements.

The extra- and intracellular $\alpha$-amylase concentrations during the culture are shown in Fig. 5(a) and (b), respectively. After $100 \mathrm{~h}$, a steady-state level in both the intra- and the extracellular concentrations was obtained. The steady-state level of the intracellular concentration was about 17 FAU (g dry wt) $)^{-1}$ and since the specific $\alpha$ amylase production rate was about $15 \mathrm{FAU}(\mathrm{g} \text { dry wt })^{-1}$ $\mathrm{h}^{-1}$, it was found that the turnover time of the intracellular $\alpha$-amylase pool was $1 \cdot 1 \mathrm{~h}$. When the glucose medium was changed to the maltose medium, the intracellular level of $\alpha$-amylase decreased rapidly, followed by an increase to approximately the same level. At the same time, the extracellular concentration increased until it reached a new steady state at $1 \cdot 2 \mathrm{FAU} \mathrm{ml} l^{-1}$. The residence time for the intracellular $\alpha$-amylase pool at the steady-state level was only about $42 \mathrm{~min}$. When the medium was changed back to glucose, the $\alpha$-amylase production instantly decreased from $25 \mathrm{FAU}$ (g dry wt) ${ }^{-1} \mathrm{~h}^{-1}$ to $16.4 \mathrm{FAU}$ (g dry wt $)^{-1} h^{-1}$. This was seen in the change in the extracellular $\alpha$-amylase concentration, which can be de-

Table 1. Physiological variables for the three $A$. oryzae strains

\begin{tabular}{|c|c|c|c|c|}
\hline Strain & $\begin{array}{c}\text { Mean } \\
\text { intracellular } \\
\text { concn [FAU (g } \\
\left.\text { dry wt })^{-1} \pm S D\right]\end{array}$ & $\begin{array}{c}\text { Specific } \\
\text { productivity } \\
\text { [FAU }(g \text { dry } \\
\left.w t)^{-1} h^{-1}\right]\end{array}$ & $\begin{array}{c}\text { Residence } \\
\text { time (min } \pm \\
\text { SD) }\end{array}$ & $\begin{array}{c}\text { Maximum } \\
\text { extracellular } \alpha- \\
\text { amylase concn } \\
\left(\text { FAU } \mathbf{~ m}^{-1} \text { ) }\right.\end{array}$ \\
\hline A1560 & $3 \cdot 5 \pm 1$ & $7 \cdot 5$ & $28 \pm 8$ & 0.6 \\
\hline CF1.1 & $15 \pm 1$ & 24 & $38 \pm 3$ & $2 \cdot 2$ \\
\hline $\mathrm{CF} 2.1$ & $17 \pm 1$ & 30 & $34 \pm 3$ & $3 \cdot 1$ \\
\hline
\end{tabular}



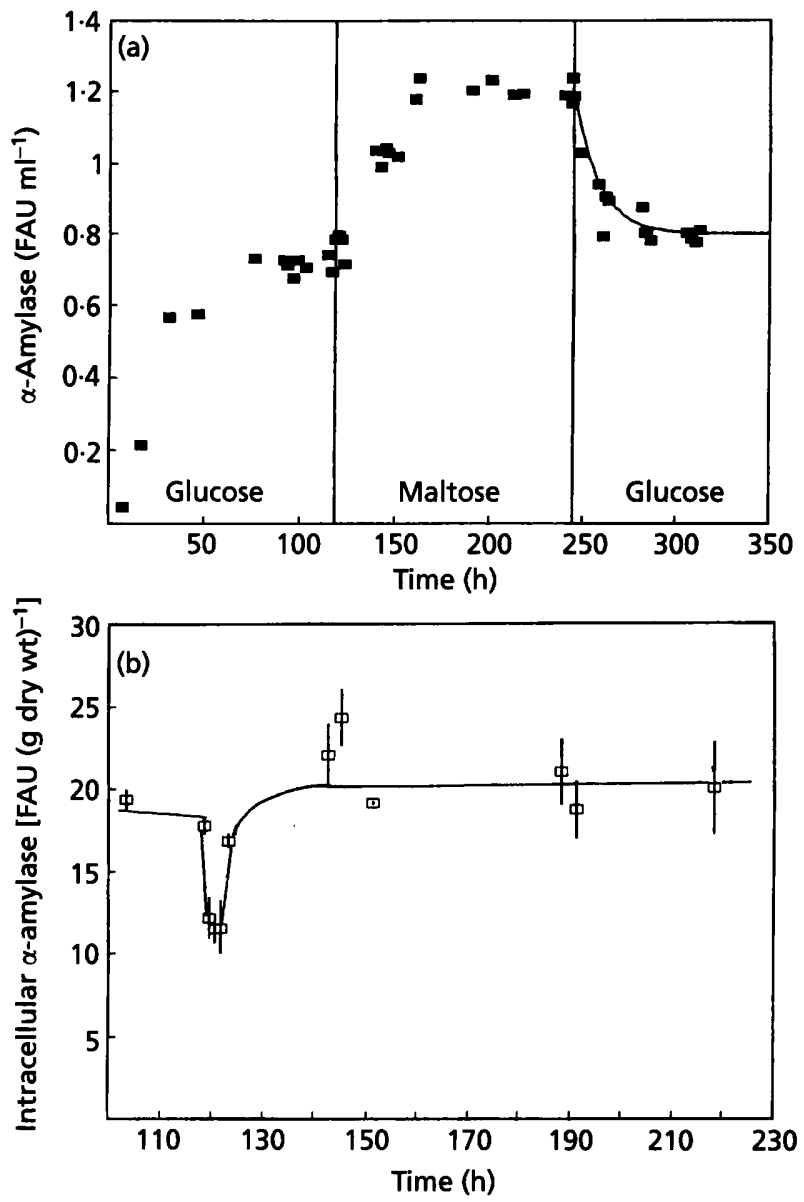

Fig. 5. (a) Chemostat experiment with A1560. After $118.75 \mathrm{~h}$, the medium was changed from a glucose medium to a maltose medium and after $245.25 \mathrm{~h}$, it was changed back to glucose, as shown. The dilution rate was $0.075 \mathrm{~h}^{-1}$. The extracellular $\alpha$ amylase concentration profile during continuous culture is shown plotted against time. The line after $245 \cdot 25 \mathrm{~h}$ represents the simulated $\alpha$-amylase concentration during a specific productivity of 16.4 FAU (g dry wt) $)^{-1} h^{-1}$. (b) The intracellular $\alpha$ amylase level during the medium change from glucose to maltose (at $118.75 \mathrm{~h}$ ) in continuous culture. The absolute SD is given as vertical error bars.

scribed as a wash-out profile using this specific production rate.

\section{DISCUSSION}

Immunoblotting, silver-staining and in situ $\alpha$-amylase activity assays of polyacrylamide gels strongly indicated that the band at $54 \mathrm{kDa}$ was $\alpha$-amylase. The intensity of this band on immunoblots was therefore used to get a quantitative value of the intracellular $\alpha$-amylase concentration. Two other bands were visualized on the immunoblot, a band at $66 \mathrm{kDa}$ and a weak band at $52 \mathrm{kDa}$. In situ activity gels showed that the $66 \mathrm{kDa}$ band has starch-degrading activity. Deglycosylation experiments indicate that the deglycosylated form of $\alpha$-amylase was the $52 \mathrm{kDa}$ band. The unglycosylated form of $\alpha$ amylase was not detected in samples from wild-type strain culture, whereas the recombinant strains, especially CF2.1, produced this unglycosylated form. This indicates that the post-translational step, glycosylation, cannot catch up with the production of the unglycosylated form at the higher production rate of the recombinant strains.

The unglycosylated form was detected both intra- and extracellularly which indicates that this form can also be transported over the cell membrane and wall. The ratio between the unglycosylated and the glycosylated form in the batch culture with the recombinant strain CF2.1 was $12 \pm 2 \%$ for the intracellular samples and $11 \pm 2 \%$ for the extracellular samples. If there is no secretion of the unglycosylated form, this would only be present extracellularly due to fragmentation and the intracellular ratio would therefore be larger than the extracellular ratio. Since the ratios were equal it seems that the glycosylation of $\alpha$-amylase is not crucial for the secretion process.

Only two types of starch-degrading enzymes have been reported in $A$. oryzae, $\alpha$-amylase and AMG, and the $66 \mathrm{kDa}$ protein is therefore strongly believed to be AMG. Recognition of AMG by antibodies could be explained by the presence of AMG in small amounts in the $\alpha$-amylase standard, which was used to immunize the rabbit.

The intracellular $\alpha$-amylase concentrations for the three strains of $A$. oryzae during batch fermentations all follow the same trend. In the exponential growth phase where the glucose concentration was high, the intracellular $\alpha$ amylase concentration was almost constant. The intracellular concentration for the recombinant strains was about four to five times higher than for the wild-type strain.

The specific productivity and the residence time for the intracellular pool were calculated for the exponential growth phase. The specific productivity for the recombinant strains was about three times higher than for the wild-type strain, which indicates that the higher intracellular concentration is not a consequence of limitations in the secretion system, but rather is caused by the higher production rate due to the additional genes encoding $\alpha$-amylase. The residence times for the intracellular $\alpha$-amylase pool were in the same range for the three strains, which supports the hypothesis that the secretion system can handle the higher productivity.

Hubbard \& Ivatt (1981) give a rough approximation of the time course of processing a glycoprotein in a eukaryotic cell to be between 30 and $60 \mathrm{~min}$, which correlates quite well with the residence time calculated for the three strains.

A drastic increase in the intracellular concentration occurred at the end of the batch cultures when the glucose was depleted, whereas the extracellular $\alpha$-amylase production continued at the same rate. The increase in the intracellular $\alpha$-amylase level is therefore not due to a reduction in the secretion process, but rather a derepression of production when glucose is depleted, resulting in increased $\alpha$-amylase production. This is supported by the glucose-limited chemostat experiments where the specific $\alpha$-amylase productivity was about two 
times higher than during the exponential growth phase where the glucose concentration was high. In the glucoselimited chemostat, the residual glucose concentration was about $2 \mathrm{mg}^{-1}$ and from other chemostat experiments it has been found that glucose concentrations below $5 \mathrm{mg}$ $1^{-1}$ do not repress $\alpha$-amylase production (Carlsen, 1994). Since there was no repression of glucose in the chemostat experiment, it is obvious that there was induction by maltose since the specific productivity increased more than $60 \%$ upon changing to a maltose medium in the chemostat. Thus, production of $\alpha$-amylase by $A$. oryzae is induced by maltose and is partly repressed by glucose, i.e. there is a constitutive production at high glucose concentrations but with a twofold higher production rate at low glucose concentrations.

The rapid decrease in the intracellular concentration compared with the increase in the extracellular $\alpha$-amylase concentration strongly indicates that there was a rapid increase in the secretion of $\alpha$-amylase caused by maltose induction. The following increase in the intracellular concentration can be explained by increased $\alpha$-amylase synthesis, which was also induced by maltose. Thus, the secretion was regulated faster than the synthesis of $\alpha$ amylase. After $150 \mathrm{~h}$, the intra- and extracellular concentration reached a steady-state level, where the production and the secretion were in balance. The significantly lower residence time for the intracellular $\alpha$-amylase pool for the maltose-limited culture, also indicates that maltose induces secretion of $\alpha$-amylase.

\section{REFERENCES}

Barbesgaard, P., Heldt-Hansen, H. P. \& Diderichsen, B. (1992). On the safety of Aspergillus oryzae: a review. Appl Microbiol Biotechnol 9 , 569-572.

Benthin, S., Nielsen, J. \& Villadsen, J. (1991). Characterization and application of precise and robust flow-injection analysers for online measurement during fermentations. Anal Chim Acta 247, 45-50.

Bhella, R. S. \& Altosaar, I. (1987). Translational control of $\alpha$ amylase gene expression in Aspergillus awamori. Biotech Appl Biochem 9, 287-293.

Carlsen, M. (1994). $\alpha$-Amylase production by Aspergillus oryzae. $\mathrm{PhD}$ thesis, Technical University of Denmark.

Carlsen, M., Marcher, J. \& Nielsen, J. (1994). An improved FIA- system for measuring $\alpha$-amylase in cultivation media. Biotech Techn 8, 479-482.

Carlsen, M., Spohr, A. B., Nielsen, J. \& Villadsen, J. (1995a). Morphology and physiology of an $\alpha$-amylase producing strain of Aspergillus oryzae during batch cultivations. Biotechnol Bioeng (in press).

Carlsen, M., Nielsen, J. \& Villadsen, J. (1995b). Kinetic studies of acid-inactivation of $\alpha$-amylase from Aspergillus oryzae. Chem Eng Sci (in press).

Christensen, T., Woldike, H., Boel, E., Mortensen, S. B., Hjortshøj, K., Thim, L. \& Hansen, M. T. (1988). High level expression of recombinant genes in Aspergillus oryzae. Bio/Technology 6, 1419-1422.

Erratt, J. A., Douglas, P. E., Moranelli, F. \& Seligy, V. L. (1984). The induction of $\alpha$-amylase by starch in Aspergillus oryzae: evidence for controlled mRNA expression. Can J Biochem Cell Biol 62, 678-690.

Fogarty, W. M. (1983). Microbial amylases. In Microbial Enzymes and Biotechnology, pp. 1-92. Edited by W. M. Fogarty. London: Applied Science Publishers.

Hansen, P. W. (1984). Determination of fungal $\alpha$-amylase by Flow Injection Analysis. Anal Cbim Acta 158, 375-377.

Hubbard, S. C. \& Ivatt, R. J. (1981). Synthesis and processing of asparagine-linked oligosaccharides. Annu Rev Biochem 50, 555-583.

Huge-Jensen, B., Andreasen, F., Christensen, T., Christensen, M., Thim, L. \& Boel, E. (1989). Rhizomucor miebei triglyceride lipase is processed and secreted from transformed Aspergillus oryzae. Lipids 24, 781-785.

Laemmli, U. K. (1970). Cleavage of structural proteins during the assembly of the head of bacteriophage T4. Nature 227, 680-685.

McKelvy, J. F. \& Lee, Y. C. (1969). Microheterogeneity of the carbohydrate group of Aspergillus oryzae $\alpha$-amylase. Arch Biochem Biopbys 132, 99-110.

Stein, E. A. \& Fischer, E. H. (1958). The resistance of $\alpha$-amylase towards proteolytic attack. $J$ Biochem Chem 232, 867-879.

Tsuchiya, K., Gomi, K., Kitamoto, K., Kumagai, C. \& Tamura, G. (1993). Secretion of calf chymosin from the filamentous fungus Aspergillus oryzae. Appl Microbiol Biotech 40, 327-332.

Ward, P. P., Lo, J.-Y., Duke, M., May, G. S., Headon, D. R. \& Conneely, O. M. (1992). Production of biologically active recombinant human lactoferrin in Aspergillus oryzae. Bio/Tecbnology 10, 784-789.

Yabuki, M., Ono, N., Hoshino, K. \& Fukui, S. (1977). Rapid induction of $\alpha$-amylase by non-growing mycelia of Aspergillus oryzae. Appl Environ Microbiol 34, 1-6.

Received 18 May 1995; accepted 23 June 1995. 